\title{
Location-Based Adaptive Detection Threshold for Dynamic Spectrum Access
}

\author{
Jad Nasreddine, Janne Riihijärvi, Petri Mähönen \\ Institute for Networked Systems, RWTH Aachen University \\ Kackertstrasse 9, D-52072 Aachen, Germany \\ Email: \{jad, jar, pma\}@mobnets.rwth-aachen.de
}

\begin{abstract}
In this paper, we propose a heuristic algorithm that exploits additional information, such as related to geo-location and frequency allocation, that can be in general known by a secondary network willing to opportunistically access the spectrum allocated to a primary network. The algorithm aims at finding the maximum allowed transmission power with which a secondary node can transmit while respecting primary constraints. It is designed to be used in the presence of multiple primary transmitters with known positions but without information about their activity patterns. The estimation of the allowed transmission power is done using a semi-analytical model characterizing interference probability. The latter is defined as the probability that the interference generated by the secondary node to any of the users in a primary cell exceeds a fixed threshold. The main idea is to autonomously determine a detection threshold based on the relative position of the secondary node towards base stations. Simulation results in a simple scenario are analyzed in order to explain the behavior of the algorithm. In addition, more results are introduced to evaluate the performance of the algorithm in more realistic scenarios.
\end{abstract}

Index Terms-Dynamic Spectrum Access, location-based detection threshold, multiple primary transmitters.

\section{INTRODUCTION}

The current approach to spectrum management has been shown to be inefficient due to its static nature. One approach to increase flexibility and enhance spectrum efficiency is to allow opportunistic access by secondary nodes to licensed spectrum. In this solution, opportunistic access would typically be allowed only if the experienced interference by any primary receiver due to the presence of secondary activity does not exceed a predefined threshold [1]. Therefore, a secondary node can transmit with a given power only if its path loss towards the closest primary receiver is high enough to reduce the incurred interference below the required threshold. In order to estimate the path loss, the distance between the secondary node and the worst case primary receiver should be known.

The computation of the path loss will depend highly on the available information and the considered use case. When there is information about neither the primary cells and the secondary nodes, transmitter detection based on spectrum sensing is the most suitable technique. This is true especially when only one primary is transmitting or the transmitters are very far apart, which is the case of TV primary networks. This problem has been extensively studied as in [2], [3]. However, the condition of having one primary transmitter is not met when we consider wireless networks where several neighboring transmitters are active and the signal sensed by the secondary node will be the sum of all their signals. In this case, different approaches are needed to solve the problem. In [4], the authors study the feasibility of opportunistic access in a frequency-planned wireless network. The main conclusion is that the secondary nodes have to transmit with very low power and should have high sensitivity. In [5], the authors propose a collaborative opportunistic access algorithm for scenarios with multiple primary transmitters. They assume that the positions of the primary base stations and their numbers are not known, but several sensing samples are considered to find the closest base station. The detection is done using a distributed database. The drawback of this method is that it requires a vast amount of data exchange.

In this paper we consider a primary network with multiple transmitters using the same frequency for downlink. Since the position of primary terminals cannot be known (due to mobility for instance), we only assume that the positions of the primary base stations as well as the position of the secondary node iteself are known. One well known method to have the information about primary base station positions is to have regional databases. This approach has been adopted by the FCC [6]. Moreover the primary network can obtain more benefits from communicating such type of information to the secondary network that will pay for the information in order to enhance its performance. In general, the base stations of a wireless network are not all active at the same time. Herein we assume that the secondary node does not know the distribution of active base stations or their activity patterns. Moreover, we assume that the secondary node cannot extract the corresponding signals for each base station. This is the case when the secondary node cannot decode the primary signals since the primary network might not be interested to communicate the required information for the decoding mechanism. Our objective is to find the maximum allowed power of the secondary node in these conditions while respecting primary constraints. In order to solve this problem, we propose a new algorithm that is based on a dynamic threshold for detecting the activity of primary base stations. For each position of a secondary node, the closest active base station ${ }^{1}$ is detected by the secondary node using the dynamic threshold. Based on

\footnotetext{
${ }^{1}$ In this paper, we use the term 'closest' to refer the cell that will experience the highest interference from the secondary node.
} 
this information, the secondary node determines the maximum power that can be used while respecting primary constraints. For the computation of the power, we consider that only one secondary node is transmitting (e.g. by using time division multiple access). For the case where different secondary nodes can transmit, any of the existing methods that have been proposed in the literature to solve the problem of multiple secondary nodes when only one primary transmitter is active (e.g. [3], [7], [8]) can be used.

Some of the regulators like OFCOM have already pointed out the need of location-dependent threshold in the context of dynamic spectrum access. This paper introduces a suitable algorithm for this new paradigm. To our best knowledge this is the first algorithm in this context. The main advantage of the proposed approach, in the context of multiple primary transmitters, is the fact that it benefits from the additional available information (i.e. location and frequency allocation) that have not been used in other approaches to increase the efficiency of spectrum usage. In addition, cooperation is not imperative as in [5], although it can increase system performance.

The rest of this paper is organized as follows. In Section II we formulate the problem and present the considered assumptions. In Section III we introduce the proposed algorithm that is evaluated in Section IV. In Section V we conclude the paper and give an overview of future work.

\section{Problem Formulation}

In this paper, we assume that the primary networks are not limited to broadcasting networks, meaning that each cell can have specific activity pattern where the allocated frequency bands to this cell are assigned to specific terminals. The activity pattern is assumed to be unknown to the secondary nodes. Therefore, the latter have to periodically scan the allocated frequency bands to the primary in order to find spectrum holes. A spectrum hole for a given secondary node is defined here as a frequency band where the node can transmit with a power higher than a threshold, $P_{\min }$, that depends on secondary architecture and properties. In this context, the paper provides a methodology that enables the secondary node to decide about its allowed transmitted power taking into account primary constraints. The detection of spectrum holes is based on transmitter detection, considering that the position of primary receivers (i.e. terminals) are unknown but the boundaries for each cell, where the terminals are enclosed, are approximated and made available for the secondary. Moreover, we assume that the secondary is interested only in spectrum holes that correspond to the periods of time where the closest primary base stations are not serving a user at the frequency of interest (i.e. inactive periods at inter-packet level are not considered here).

We consider a wireless system with $F$ frequency bands that are distributed over the different cells. Moreover, we consider that each frequency band can be allocated to only one user in a given cell. This is equivalent to a chunk in an OFDMA-based system [9].

\section{A. Primary Constraints}

In order to allow opportunistic access, the primary network should tolerate loss in service for some of its users or an increase in the transmitted power [2]. The loss in service can be either reflected by a reduction of the coverage area [4] or by an acceptable interference probability [10], [11]. In this paper, we use the latter ${ }^{2}$. The interference probability with respect to a primary user, $p$, is defined as the probability that the experienced interference $I$ by $p$ due to secondary activity exceeds a predefined threshold $\iota_{\max }$ :

$$
\operatorname{Pr}_{\text {int }} \triangleq \operatorname{Pr}\left\{I \geq \iota_{\max }\right\} .
$$

The primary network will specify an interference constraint that will ensure that all its users have the required Quality of Service (QoS), meaning that the outage probability is kept at a low level. This is reflected by the fact that the interference probability $\operatorname{Pr}_{\text {int }}$ experienced by any primary terminal should not exceed a threshold $\varepsilon$.

In this paper, we assume that any required approximation should be biased to a more conservative approach with respect to primary protection. Since the positions of the primary terminals are not known to the secondary nodes (in addition to mobility issues), the worst case situation is considered. This means that the secondary node should guarantee that an active primary terminal that might be at the position where the interference generated by secondary activity is at maximum will be satisfied (i.e., $\operatorname{Pr}_{\text {int }}<\varepsilon$ ). Therefore, the secondary node will consider the so-called Worst Case primary terminal Position (WCP) for its power decision. The WCP is the closest possible primary terminal position in the primary coverage areas that have the lowest mean path loss toward the secondary node. However, the coverage areas of wireless network cells do not, in general, have a known and regular shape. Therefore the secondary will consider the circle that contains the coverage area as shown in Figure 1, which is again a conservative assumption to protect the primary. This circle will be considered in the following as the estimated coverage area.

In our algorithm, we consider that the WCP is the intersection of the estimated primary coverage area and the line connecting the primary and the secondary transmitters. This is true when we consider a monotonic distance-dependent path loss where the shadow fading is considered separately in the computation. This conservative approach is chosen in order to protect primary users especially in the scenarios where no information about the shape of the coverage area is available. However, if such information is available, as in the case of the implementation of a Radio Environment Map (REM) [12], more precise estimation of the WCP can be implemented.

A special case appears, when the secondary is inside the estimated coverage area of an active primary base station. In this case, the secondary is forbidden to transmit since a primary terminal can be extremely close to him.

\footnotetext{
${ }^{2} \mathrm{~A}$ mapping between the two approaches can be derived when specific primary networks with known system characteristics is considered.
} 


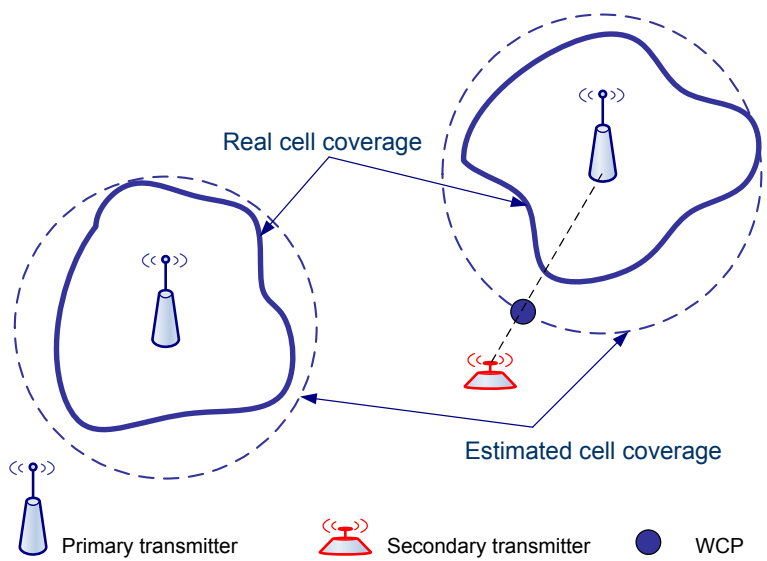

Fig. 1. Estimated Coverage Areas and Worst Case primary terminal Position (WCP).

The derivation of the values of $\iota_{\max }$ and $\varepsilon$ depends on system characteristics and can be performed using a planning tool, which can be either static or dynamic.

It should be noted that when our conservative approach is considered, the interference probability is computed for an extreme position of the primary terminal (i.e., the WCP). In general, the probability that a primary terminal is at this position is very low, which leads to a highly protective scheme for primary users. This is unavoidable assumption in case we do not know the positions of the primary terminals that should be protected in order that primary networks allow spectrum sharing.

\section{B. Available Information and Assumptions}

We assume that the secondary node knows its own position. Moreover, for the sake of simplicity we assume that all the antennas are omnidirectional and that the propagation model is homogeneous with log-normal shadowing ${ }^{3}$ [10]. Finally, we consider in this paper that the transmission power of the primary is fixed and we only consider the downlink.

We consider that the collaboration between primary and secondary networks is performed on a long-term scale and is determined based on the following data:

- The positions of primary base stations, the estimated coverage and their transmit powers are communicated to the secondary network. This can be done either using a common control channel, such as proposed in IEEE P1900.4 [13], that directly communicates the information to the secondary node or using a fixed link between primary and secondary networks.

- The secondary network respects the constraint on the experienced interference by primary users due to its activity.

\footnotetext{
${ }^{3}$ In reality an uncertainty model should be considered along with the propagation model as in [2]. However, we will not consider the uncertainty model in this paper for simplicity.
}

- In case that the primary performs long-term frequency planning, the frequency allocation to primary cells can be optionally communicated to the secondary network.

In the description of the algorithm we differentiate between cells that are associated to a frequency $f$, and active cells using this frequency. The former are not necessarily transmitting while the latter have a primary terminal to which the base station is transmitting data. We denote by $\Phi_{f}$ and $\Pi_{T}$ the set of cells that are associated to frequency $f$ and the set of all transmitting primary base stations, respectively.

The power of the received signal at the secondary node, $c$, due to all transmitting primary base stations is given by

$$
R_{c}=10 \log _{10}\left(\sum_{j \in \Pi_{T}} 10^{R_{c j} / 10}\right),
$$

where $R_{c j}$ is the received power in $\mathrm{dBW}$ by the node $c$ due to the transmission of primary base station $j$ given by

$$
R_{c j}=P_{j}-L_{c j}\left(d_{c j}\right)+\xi_{c j},
$$

where $P_{j}$ is the transmitted power by the base station $j$, $L_{c j}\left(d_{c j}\right)$ is the path loss between the primary base station $j$ and the secondary node $c$ that are separated by distance $d_{c j}$ and $\xi_{c j} \sim \mathcal{N}(0, \sigma)$ is the normal shadowing factor with zero mean and standard deviation $\sigma$. Moreover, we do not consider fast fading since it occurs in smaller time scale than shadowing and can be eliminated by averaging [14].

\section{Secondary Objectives and Metrics}

Secondary networks aim in general at maximizing their throughput using the released frequency bands by the primary network. Throughput maximization depends on the following factors:

- The number of released frequency bands.

- The surface and the shape of the region where the released frequencies can be used.

- The allowed power with which the secondary node can transmit while satisfying primary constraints on interference.

In order to reflect these factors, we represent the efficiency of the spectrum detection method by the histograms of the allowed transmitted power and a new metric that represents the contiguous zones where the secondary node can transmit using a power higher than threshold $P_{\min }$ either using all the available frequency bands or using the frequency band where the highest power is allowed. This new metric is called Useful Reused Surface (URS) and it is inspired by the metric proposed in [15] to reflect the efficiency of spectrum management mechanisms in primary networks. The URS is defined here as

$$
\mathrm{URS}=\sum_{c=1}^{C} S_{c} \omega_{c},
$$

where $C$ is the set of non-contiguous areas where the primary frequencies can be used by the secondary node, $S_{c}$ is the surface of the contiguous area $c$ in $\mathrm{km}^{2}$ and $\omega_{c}$ is a weight 
that depends on the expected number of secondary users in this area to account for the fact that the release of carriers will be more effective in areas with a significant number of potential secondary users. Here we set simply $\omega_{c}=S_{c} / S$, where $S$ is the total surface of the system.

\section{Spectrum Detection Algorithm BASED ON ADAPTIVE THRESHOLD}

In the Spectrum Detection Algorithm based on Adaptive Threshold (SDA-AT), the secondary node exploits the available information concerning its position in addition to primary characteristics and constraints.

\section{A. Main Idea}

The main idea is to determine the maximum power $P_{S}$, with which secondary node $c$ can transmit at a given frequency while keeping the probability of interference toward each primary cell lower than the allowed threshold. Since the introduced interference to a primary cell is highly correlated to the distance towards the secondary node, the detection of the closest active cell to the latter is a key enabler to compute $P_{S}$. This interference depends also on the shadow factor that is characterized by its log-normal distribution. Therefore $P_{S}$ will not only depend on the detection of the active base station but also on the estimation of introduced interference when this base station is detected.

When estimating its interference probability with respect to an active primary cell $j$, a secondary node should consider the following two complementary events:

$\bar{M} \quad$ The activity of the cell is detected and the secondary node can transmit with power $P_{S D}(j) \in\left[0, P_{\max }\right]$, where $P_{\max }$ is the maximum possible power due to the limitations of the secondary node. Power $P_{S D}(j)$ is equal to 0 when the secondary node is inside the estimated coverage area of cell $j$.

$M \quad$ The activity of the cell is not detected and the secondary node can transmit with higher power $P_{S M}(j) \in\left(0, P_{\max }\right]$.

The estimated interference probability $\operatorname{Pr}_{\text {int }}$ at the Worst Case primary terminal Position (WCP) $p$ in cell $j$ can be written according to the law of total probability as

$$
\begin{aligned}
\operatorname{Pr}_{\text {int }}= & \operatorname{Pr}\left\{I \geq \iota_{\max } \mid M\right\} \operatorname{Pr}\{M\} \\
& +\operatorname{Pr}\left\{I \geq \iota_{\max } \mid \bar{M}\right\} \operatorname{Pr}\{\bar{M}\} .
\end{aligned}
$$

In the rest of this paper, probabilities $\operatorname{Pr}\left\{I \geq \iota_{\max } \mid \bar{M}\right\}$ and $\operatorname{Pr}\left\{I \geq \iota_{\max } \mid M\right\}$ are called probabilities of high interference with detection and without detection and denoted by $\operatorname{Pr}_{h d}(j)$ and $\operatorname{Pr}_{h m}(j)$, respectively, where $j$ is the considered primary cell. Moreover, the probability that event $M$ occurs is called probability of miss-detection $\operatorname{Pr}_{m}(j)$, and we have

$$
\operatorname{Pr}\{\bar{M}\}=1-\operatorname{Pr}_{m}(j) .
$$

Combining (5) and (6) and taking into account that at the limit $\operatorname{Pr}_{\text {int }}$ should be equal to $\varepsilon$, we obtain

$$
\operatorname{Pr}_{m}(j)\left[\operatorname{Pr}_{h m}(j)-\operatorname{Pr}_{h d}(j)\right]+\operatorname{Pr}_{h d}(j)=\varepsilon .
$$

The detection of the base station can be done using different techniques. In this paper, we consider a detection methodology based on the comparison of the total received power $R_{c}$ with a dynamic threshold $R_{\mathrm{th}}(j)$. If $R_{c}$ is higher than $R_{\mathrm{th}}(j)$, the base station $j$ is assumed to be active. In this case, the probability of miss-detection $\operatorname{Pr}_{\mathrm{m}}(j)$ (i.e., the probability that $R_{c}$ is lower $R_{\text {th }}(j)$ while $j$ is active) is

$$
\begin{aligned}
\operatorname{Pr}\{M\} & =\operatorname{Pr}_{\mathrm{m}}(j) \\
& =\operatorname{Pr}\left\{R_{c} \leq R_{\mathrm{th}}(j) \mid j \text { is active }\right\} .
\end{aligned}
$$

In general, $R_{c}$ is the summation of the total received power from all the active cells. Therefore, $R_{c}$ is always higher than any component $R_{c j}$. Hence, we can write

$$
\operatorname{Pr}_{\mathrm{m}}(j) \leq \operatorname{Pr}\left\{R_{c j} \leq R_{\mathrm{th}}(j) \mid j \text { is active }\right\} \forall j .
$$

This upper-bound will be used as a conservative approximation in this paper. In this context the system becomes more conservative when the contribution of the other base stations than the tested one becomes higher. By using this conservative approach, we increase the protection for primary users but we reduce the opportunities for secondary communication. However, we consider this approach as a first solution to the problem.

Since we consider a path loss that depends on the distance and the shadow fading, which is a log normal random variable, the miss-detection probability can be written using (3) and the upper bound in (9) as

$$
\operatorname{Pr}_{\mathrm{m}}(j)=\frac{1}{2}\left[1+\operatorname{erf}\left(\frac{R_{\mathrm{th}}(j)-P_{j}+L_{c j}\left(d_{c j}\right)}{\sigma \sqrt{2}}\right)\right] .
$$

Since we consider that the position of the primary terminal cannot be known by the secondary nodes, two scenarios appears depending on the relative position of the secondary node with respect to the closest cell $j$ :

- The secondary node is inside the estimated coverage area of the cell. In this case, the secondary node is forbidden to transmit if the corresponding base station is active. Otherwise, its transmitted power is highly correlated to its relative distance with respect to the next cell. Therefore, probabilities $\operatorname{Pr}_{h d}(j)$ and $\operatorname{Pr}_{h m}(j)$ are equal to zero and unity, respectively.

- The secondary node is outside the estimated coverage area of the active base station. In this case the secondary node can always transmit but its power is highly correlated to its relative distance with respect to the closest active cell.

In the first case, the interference constraint corresponding to cell $j$ becomes

$$
\operatorname{Pr}_{\mathrm{m}}(j)=\varepsilon \text {. }
$$

Therefore, the value of $R_{\mathrm{th}}(j)$ can be written as

$$
R_{\mathrm{th}}(j)=\operatorname{erf}^{-1}(2 \varepsilon-1) \sigma \sqrt{2}+P_{j}-L_{c j}\left(d_{c j}\right) .
$$

As we can see in (12), the threshold depends on the transmitted power of the primary, the path loss components 
and the distance between the secondary node and the primary base station.

A secondary node can be in the coverage area of different primary base stations that are assumed by the secondary node to be associated to frequency $f$. The set of these base stations is denoted here by $C_{f}$. The set $C_{f}$ is built by the secondary node using the available information. Therefore it can include all cells in $\Phi_{f}$ or only the active ones. For each base station $j$ in $C_{f}$, the secondary node compares the aggregate received power $R_{c}$ with dynamic power threshold $R_{\mathrm{th}}(j)$.

If at least one cell in $C_{f}$ is active (i.e. $\exists j \in C_{f}, R_{c}>$ $R_{\mathrm{th}}(j)$ ), no transmission should be allowed since a primary terminal can be anywhere inside the coverage area. In order to detect active cells, the secondary node uses an eliminatory process in which the closest base station in $C_{f}$ is checked and eliminated from the set if no activity is detected. Then the process is repeated until the set is empty.

If no active cells in $C_{f}$ are detected, the secondary node starts the process of determining its allowed transmission power $P_{S}$. The first step to determine $P_{S}$ is to find the closest active cell that does not cover the secondary node. The main problem here is the computation of $\operatorname{Pr}_{h d}(j)$ and $\operatorname{Pr}_{h m}(j)$. These are the probabilities that the interference generated by the secondary node is higher than the allowed level when the primary base station is detected or miss-detected. In the first case the secondary node will transmit using power $P_{S D}(j)$ whereas in the second case it will transmit using power $P_{S M}(j)$, yielding

$$
\operatorname{Pr}_{h d}(j)=\operatorname{Pr}\left\{P_{S D}(j)-L_{c p}\left(d_{c j}-R\right)+\xi_{c p}>\iota_{\max }\right\}
$$

and

$$
\operatorname{Pr}_{h m}(j)=\operatorname{Pr}\left\{P_{S M}(j)-L_{c p}\left(d_{c j}-R\right)+\xi_{c p}>\iota_{\max }\right\} .
$$

By considering that the shadowing factor $\xi_{c p}$ follows a normal distribution $\mathcal{N}(0, \sigma)$, we can write

$$
\operatorname{Pr}_{h d}(j)=\frac{1}{2}\left[1-\operatorname{erf}\left(\frac{\iota_{\max }-P_{S D}(j)+L_{c p}\left(d_{c j}-R\right)}{\sigma \sqrt{2}}\right)\right]
$$

and

$\operatorname{Pr}_{h m}(j)=\frac{1}{2}\left[1-\operatorname{erf}\left(\frac{\iota_{\max }-P_{S M}(j)+L_{c p}\left(d_{c j}-R\right)}{\sigma \sqrt{2}}\right)\right]$,

where $L_{c p}\left(d_{c j}-R\right)$ is the path loss between the secondary node $c$ and the WCP $p$.

Since $P_{S M}(j)$ is always higher than $P_{S D}(j)$ and the probability of high interference is an increasing function of the power, $\operatorname{Pr}_{h d}(j)$ should be strictly lower than $\varepsilon$ in order to ensure that $\operatorname{Pr}_{\text {int }}$ can be lower than $\varepsilon$ based on (7). Otherwise $\operatorname{Pr}_{h m}(j)$ should be zero and the secondary node cannot transmit with higher power than the one allowed by the interference constraint with respect to the closest non-covering cell, leading to lower use of the spectrum holes. It should be noted that increasing $R_{\mathrm{th}}$ increases the probability of detecting a spectrum hole, while increasing $P_{S D}$ and $P_{S M}$ increases the spectrum efficiency of the secondary network when a spectrum hole is detected. Therefore our objective is to find the highest values of $P_{S D}, P_{S M}$ and $R_{\mathrm{th}}$ for each base station that respect the interference constraint. When the closest cell $j$ is missdetected, the allowed power will be the one considering that the next closest cell is detected (i.e., $P_{S D}(j+1)$ ). Therefore, $P_{S M}(j)$ for the closest cell $j$ is the same as $P_{S D}(j+1)$ of the next closest cell. Hence, we can write $\left|\Phi_{f}\right|$ constraints like (7) with $\left|\Phi_{f}\right|$ power variables ${ }^{4}$ and $\left|\Phi_{f}\right|$ threshold variables. Thus we obtain a multi-objective optimization problem with non-linear constraints.

To obtain a solution we propose a heuristic algorithm where we consider that the transmitted power when a base station is detected is chosen to give a probability of high interference with detection lower than $\varepsilon$. Therefore, we can write

$$
P_{S D}(j)=\tau\left[\iota_{\max }+L_{c p}\left(d_{c j}-R\right)+\operatorname{erf}^{-1}(2 \varepsilon-1) \sigma \sqrt{2}\right],
$$

where $\tau \in(0,1)$ is a parameter that is fixed by the secondary operator and is the same for all primary cells. Depending on the value of $\tau$ the values of $P_{S D}$ and $R_{\mathrm{th}}$ will change. When $\tau$ increases the value of $P_{S D}$ will increase while the value of $R_{t h}$ will decrease. Therefore a tradeoff should be considered between the high probability of inactivity detection and the high power in the case of false detection of activity. In order to take this tradeoff into account, the activity pattern of the primary base stations should be known by the secondary node. This aspect is not included in this paper where we consider a fixed value of $\tau$.

From (7), (14) and (15), the threshold $R_{\mathrm{th}}(j)$ can be computed considering the threshold $\varepsilon$ :

$$
\begin{aligned}
R_{\mathrm{th}}(j)= & \operatorname{erf}^{-1}\left(2 \frac{\varepsilon-\operatorname{Pr}_{h d}(j)}{\operatorname{Pr}_{\mathrm{hm}}(j)-\operatorname{Pr}_{h d}(j)}-1\right) \sigma \sqrt{2} \\
& +P_{j}-L_{c j}\left(d_{c j}\right) .
\end{aligned}
$$

It should be noted that when $\operatorname{Pr}_{\mathrm{hm}}$ is lower than $\varepsilon$, the interference probability will be always lower than $\varepsilon$ since $\operatorname{Pr}_{m}(j)$ is lower than unity. In this case the secondary node will assume that the base station is not active. This happens normally when $P_{M D}(j)$ is lower than $P_{S D}(j) / \tau$.

\section{B. Algorithm Description}

Based on the available information to the secondary node concerning the frequency allocation, we have two cases:

- Primary frequency allocation is not known by the secondary node. In this case, the set $\Phi_{f}$ of cells that should be tested include all the cells in the system for each frequency $f$. In this case the algorithm is called simply SDA-AT.

- Primary frequency allocation is communicated to the secondary node. Therefore, the set $\Phi_{f}$ only contains the cells that are associated to frequency $f$ by the primary network.

\footnotetext{
${ }^{4}$ We consider that the power corresponding to the case of miss-detection of the last base station in the system to be equal to $P_{\max }$ since no base stations have been detected to be active.
} 
In this case the algorithm is called Enhanced SDAAT (ESDA-AT). This algorithm has more information about the primary network than SDA-AT and therefore its performance will be better as will be shown in the simulation section.

A high level flowchart of the algorithm is depicted in Figure 2. The secondary node scans frequency $f$ and measures the total received power $R_{c}$, which is the sum of the powers of all active base stations in the selected frequency. From the available information, the secondary node builds the set $\Phi_{f}$ of cells that are associated to frequency $f$. These base stations can be active (i.e. a primary user is active in these cells using frequency $f$ ) or idle. Then the algorithm will search for the closest active cell from set $\Phi_{f}$ with a margin of error in the interference probability that should not exceed threshold $\varepsilon$, which is fixed by the primary.

The first important step in the algorithm is to determine if any of the cells that cover the secondary node are active using frequency $f$. If this is the case the secondary node cannot use frequency $f$ for its transmission because a primary terminal can be very close to the secondary node in the coverage area. Therefore, the secondary node builds the set $C_{f} \subseteq \Phi_{f}$ which includes all cells covering the secondary node and are assumed to be associated to frequency $f$. If $C_{f}$ is not empty the secondary node will check the activity of each base station in this set starting from the closest one. In order to check if base station $j$ is active using frequency $f$, the secondary node determines the dynamic threshold $R_{\mathrm{th}}(j)$ corresponding to this cell using (12). If one cell is detected to be active, no transmission is allowed. Otherwise, the secondary node will try to identify the closest active non-covering cell and determine the allowed transmission power.

When $C_{f}$ is detected to be empty, the secondary node searches for the closest active cell in $\Phi_{f} \backslash C_{f}$. Starting from the closest cell in the latter set, the secondary node computes $R_{\mathrm{th}}$ using (16) for all cells in $\Phi_{f} \backslash C_{f}$ that have been already detected to be inactive in addition to the closest cell that have not yet been tested. The set of these cells is denoted by $\Phi_{f, t}$. This is repeated each time since the power $P_{S M}$ will change in each step leading to changes in $R_{\mathrm{th}}$ for the already tested cells. In addition $R_{\mathrm{th}}$ is not monotonic with respect to the distance. Therefore the required minimum value is not always associated to the same cell. Hence in each step the secondary node sets $R_{\mathrm{th}, \min }=\min _{j \in \Phi_{f, t}} R_{\mathrm{th}}$. If $R_{c}$ is lower than $R_{\mathrm{th}, \min }$, all cells in $\Phi_{f, t}$ are assumed to experience a reasonable interference with probability $\varepsilon$ when $P_{S M}$ is used and the same procedure is repeated for the next closest cell. Otherwise, the power $P_{S}$ is set to the highest value of $P_{S D}$. If this power is higher than $P_{\min }$, the secondary node will use the corresponding frequency to communicate. Otherwise, no transmission is allowed. $P_{\min }$ is the minimum power with which a secondary node can transmit due to hardware limitations.

The process is repeated for each frequency that the secondary node plans to scan. Then, the secondary node chooses one or several of the frequencies where transmission is allowed depending on its transmission strategy and capabilities.

In order to have a reference algorithm, we consider the ideal algorithm where the secondary node knows which are the active primary base stations.

\section{Simulations And Results}

First, we evaluate the performance of the algorithm in a simple scenario with seven hexagonal primary cells representing the seven cells that use the same frequency in a cellular system with frequency reuse of 7 . Cell radius is set to be $1 \mathrm{~km}$ and thus the base station separation distance is given by $\sqrt{3 n} R$, where $n$ is the frequency reuse factor and $R$ is the cell radius [16]. Moreover, we assume that the central cell is not active while the other six cells are transmitting with $30 \mathrm{dBm}$. This example is introduced in order to illustrate the behavior of the algorithm. In the simulations, we use the Xia-Bertoni propagation model [17], [18]. This model is chosen since it is able to take into account all types of propagation losses such as the one between base stations and the one between mobiles in addition to usual propagation loss between a base station and a mobile. Given a frequency $f$ in $\mathrm{GHz}$ and distance $d_{X Y}$ between transmitter $X$ and receiver $Y$, path loss $L_{X Y}$ is given by

$$
\begin{aligned}
L_{X Y}\left(d_{X Y}\right)= & K_{X Y}+\beta_{X Y} \log _{10}(f) \\
& +\alpha_{X Y} \log _{10}\left(d_{X Y}\right),
\end{aligned}
$$

where $K_{X Y}, \beta_{X Y}$ and $\alpha_{X Y}$ are constants computed using the Xia-Bertoni model. The obtained propagation constants are collected in Table I, where BS stands for a primary base station, SN for a secondary node and PT for a primary terminal. The antenna heights of the $\mathrm{BS}, \mathrm{CN}$ and $\mathrm{PT}$ are assumed to be equal to $27 \mathrm{~m}, 3 \mathrm{~m}$ and $1.5 \mathrm{~m}$, respectively. We consider a log-normal shadow fading with zero mean and a standard deviation of $7 \mathrm{~dB}$.

In all simulations, we consider that thresholds $\tau, \iota_{p}$ and $\varepsilon$ are equal to $0.9,-100 \mathrm{dBm}$ and 0.05 , respectively.

Figures 3 (a) and (b) represent the power distribution over the studied area when the ideal and the ESDA-AT algorithms are used. Moreover, Figures 3 (c) and (d) show the difference between the allowed power by the ESDA-AT and the ideal algorithms in addition to the CDF of this difference. In these figures we do not show the difference in the coverage of the active cells since the ideal algorithm will always forbid the secondary nodes to transmit in these zones. The first important observation from Figure 3(d) is that in more than $70 \%$ of the cases where the secondary node is allowed to transmit the gain in power by the ESDA-AT is higher than $-0.5 \mathrm{~dB}$. This means that in most cases the ESDA-AT gives similar

TABLE I

CONSTANTS OF THE PROPAGATION MODEL.

\begin{tabular}{llll}
\hline & $\alpha(\mathrm{dB})$ & $\beta(\mathrm{dB})$ & $K(\mathrm{~dB})$ \\
\hline BS - SN & 37.6 & 21 & 113.2 \\
SN - PT & 37.6 & 21 & 122.1 \\
\hline
\end{tabular}




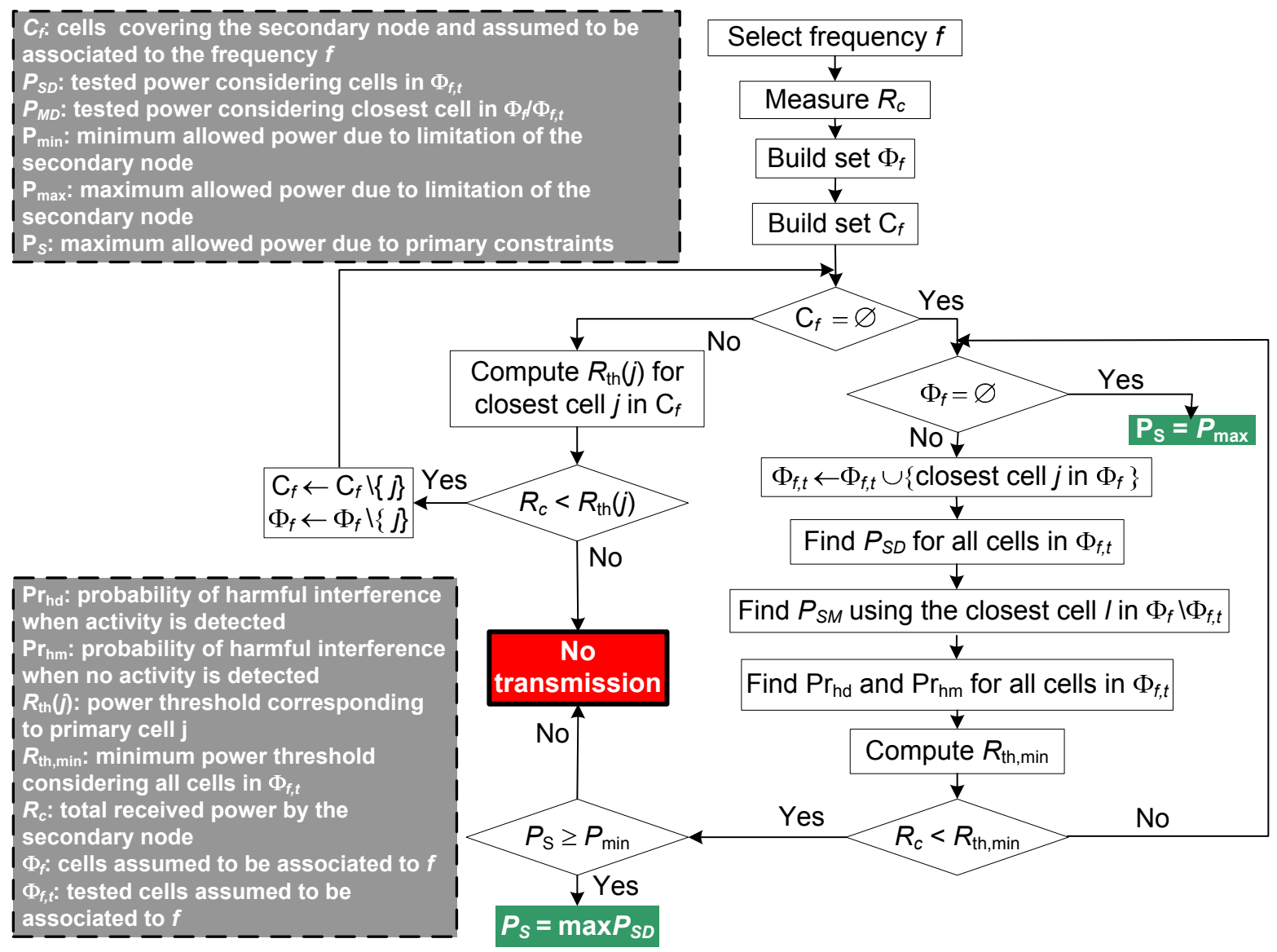

Fig. 2. High level flow chart for the (E)SDA-AT.
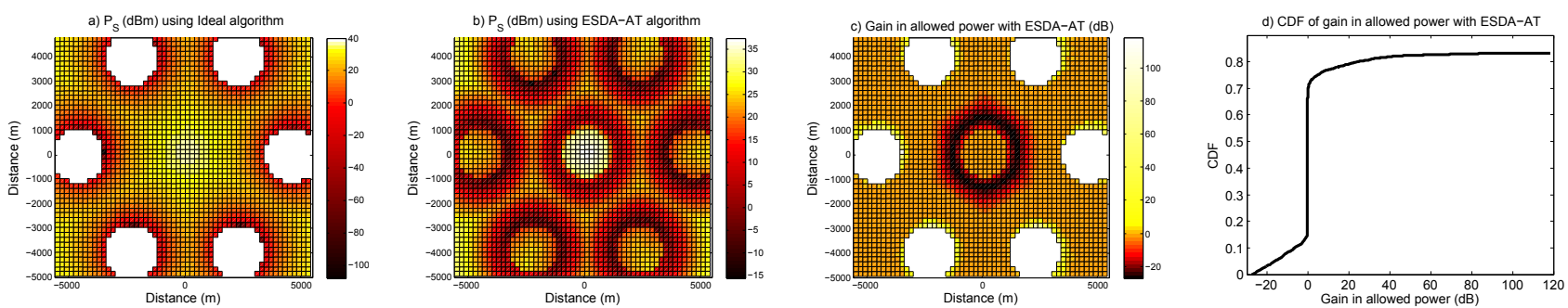

Fig. 3. Example of the power distribution and the gain with respect to the allowed power when comparing ESDA-AT to the ideal algorithm for a scenario with hexagonal cells and reuse factor of 7 (The white non-hashed zones in (a) and (c) are the zones where no transmission is allowed by the ideal algorithm).

results to the ideal algorithm in this simple example. Moreover we can see that the maximum power in the whole scenario is $30 \mathrm{dBm}$ when using the ESDA-AT, which is only $5 \mathrm{~dB}$ less than when we are using the ideal algorithm. The highest gains are obtained in the surrounding borders of the active cells. These are the only zones where the gain is positive. This is due to the fact that in the ideal algorithm the power is always estimated assuming that the active cell is the closest one. However, the activities of these cells are miss-detected in some cases when the ESDA-AT algorithm is used leading to higher allowed power. Moreover, the probability of missdetection starts to drop after this zone since the powers of the other active cells, which are not taken into account in the model of computing $R_{\mathrm{th}}$, starts to be higher making the algorithm more conservative in terms of transmitted power.

In the central cell the gain decreases when we move further away from the base station. This is due to the increased interference coming from the other cells, which leads to more conservative behavior of the algorithm as explained in subsection III-A. It should be noted that the gain decreases drastically when the borders of the coverage area are crossed. This is due to the fact that inside the coverage area we use (12) whereas outside this zone we use (16), which is more conservative. Therefore the lowest gain appears in the 
surrounding of the coverage area of the base station. The average gain starts to increase again since the allowed power when considering the central base station is active increase with distance. In all the considered positions of the secondary node the probability of interference was always equal or lower than threshold $\varepsilon$.

We shall next study the distribution of the resulting transmit powers for the secondary nodes running the two versions of the algorithm. The evaluation is performed using two scenarios: LA scenario and random scenario. In the LA scenario, the base stations are distributed in a selected area of $400 \mathrm{~km}^{2}$ in Los Angeles city based on the location data from the TMobile network [19]. In the random scenario, the base stations are randomly distributed over the same area with a minimum distance of $1 \mathrm{~km}$ between different stations.

In each scenario, we consider that three frequency bands are available for the primary network. The distribution of the frequency bands over the base stations is optimized in order to minimize the total received interference in the system, where each base station can be associated to one band. The coverage areas of the base stations are optimized in order to have a full coverage in the studied area. Moreover, we consider that primary transmission power is $30 \mathrm{dBm}$. The results are obtained based on 100 simulations with different shadowing factors. In all scenarios, the interference probability was lower or equal to $\varepsilon$, which satisfies primary constraints.

Figure 4(b) shows the histogram of resulting transmit powers summed over all the primary user channels for the LA scenario for secondary nodes running ESDA-AT. Compared to the ideal case depicted in Figure 4(c) we see that some transmission opportunities are lost, but for most locations the secondary nodes obtain transmit powers that are comparable to, for example, current Wi-Fi technologies operating on the $2.4 \mathrm{GHz}$ ISM band. For the case of SDA-AT, the performance depicted in 4(a) is understandably worse, with no transmission opportunities at roughly $40 \%$ of locations, and overall reduced transmit power. These results clearly show that the availability of frequency planning information significantly improves the performance of the algorithm. Limiting the transmissions of the secondary node to a single primary user channel allowing largest transmission power to be used does not significantly change the results, as can be seen from Figures 5(a)-5(c). Since the two cases are almost indistinguishable we shall focus on results for the total power in the following.

Figure 6(b) shows the transmit powers resulting from ESDA-AT for the random scenario with minimum distance between base stations constrained to be at least one kilometer. Overall the performance is similar to the more realistic LA case, although there is a slight decrease in transmission opportunities. Similar trend can be seen for the ideal case, as shown in Figure 6(c). The largest difference to the LA scenario arises for the SDA-AT algorithm, for which even fewer transmission opportunities arise as illustrated in Figure 6(a). For the discovered spectrum holes the distribution of transmit powers is very similar for both scenarios.

In Figures 7(a)-7(b), we present the box plot of the URS

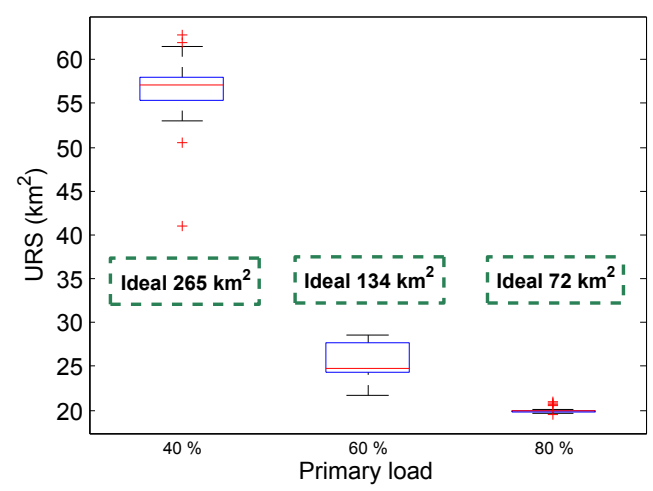

(a)

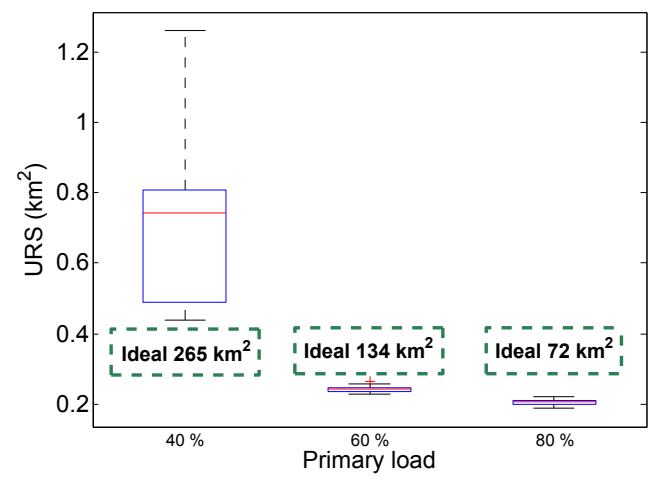

(b)

Fig. 7. The box plot of the resulting URS that corresponds to the maximum allowed power over all primary user channels for LA scenario when (a) ESDAAT is used and (b) SDA-AT is used. The values in the dashed boxes are the average values when the ideal algorithm is used.

when the ESDA-AT and SDA-AT are used respectively, where the central mark is the median, the edges of the box are the 25th and 75th percentiles, the whiskers extend to the most extreme data points not considered outliers, and outliers are plotted individually. The URS is plotted as a function of the primary load which reflects the percentage of time a base station would be active. Moreover, the URS given by the ideal algorithm is depicted inside the dashed boxes since all simulation gives the same value (the ideal algorithm results are independent of the shadow factors). The minimum allowed power $P_{\min }$ considered in this scenario is $10 \mathrm{dBm}$. In these figures we can notice that even when the SDA-AT is used the URS can be of the order of few hundreds $\mathrm{m}^{2}$ which allow an easy deployment of local access networks such as WiFi even when the load of the primary is $90 \%$. Moreover the URS decreases in an exponential way with primary load and this decay is faster in the case of ESDA-AT than in the SDA-AT.

\section{CONCLUSION}

In this paper, we have introduced the first algorithm based on location-dependent detection threshold in the context of dynamic spectrum access. The secondary node exploits the information about its position and the positions of primary base stations to detect the active cells and determine its allowed transmit power. The proposed algorithm is based on a semi- 


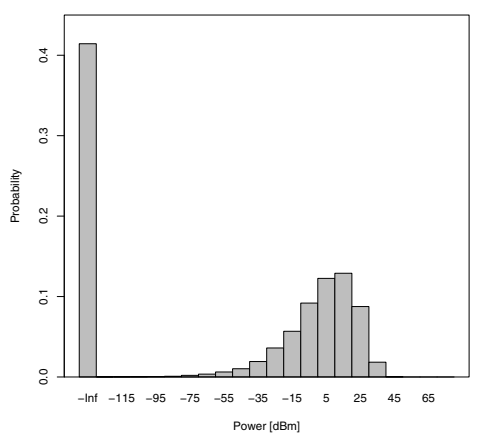

(a)

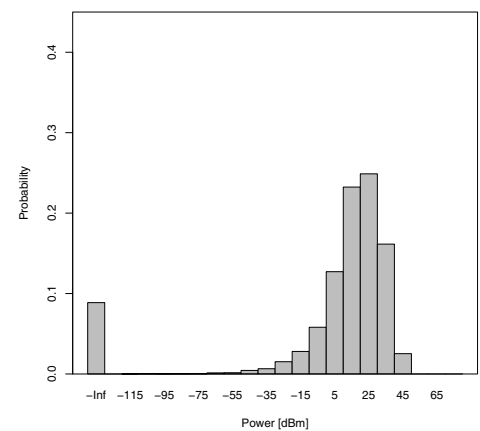

(b)

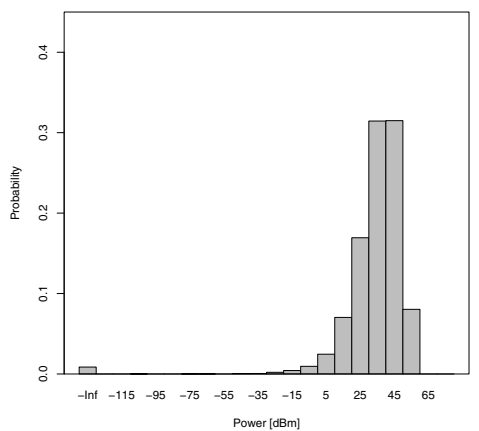

(c)

Fig. 4. The histograms of resulting transmit power summed over all the primary user channels for the LA scenario when (a) SDA-AT is used, (b) ESDA-AT is used and (c) ideal algorithm is used.

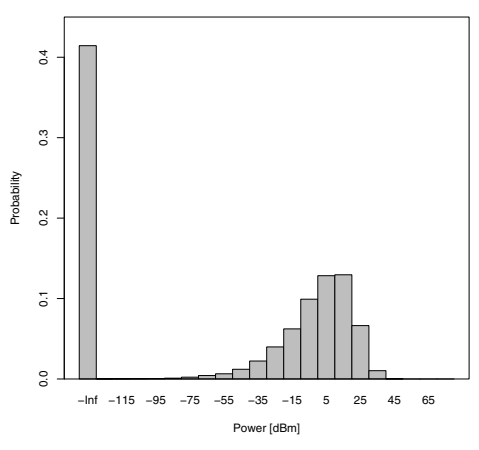

(a)

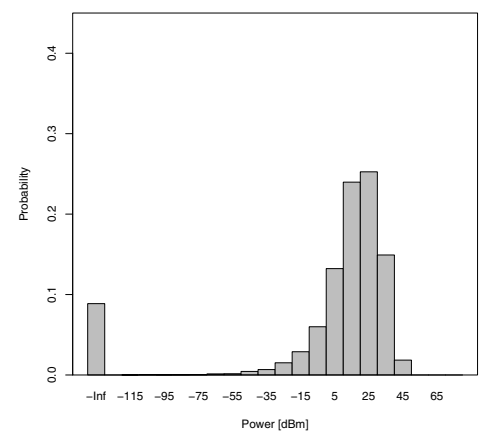

(b)

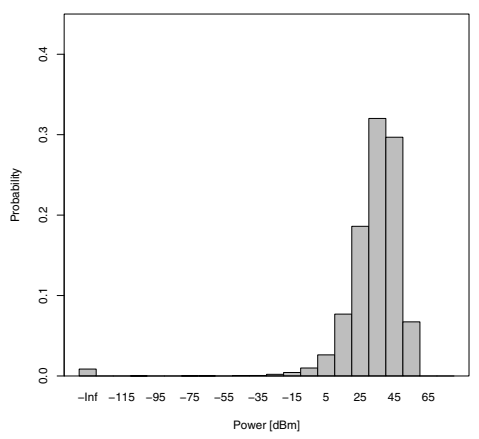

(c)

Fig. 5. The histograms of resulting maximum transmit power over all the primary user channels for the LA scenario.

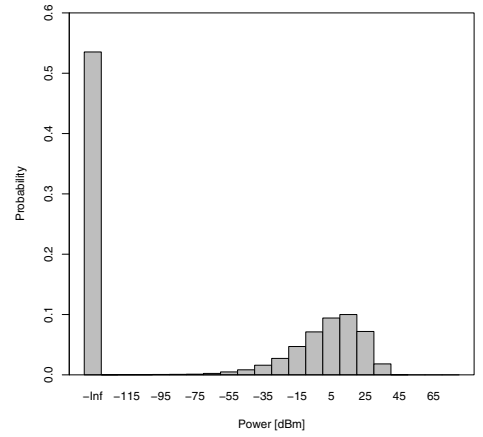

(a)

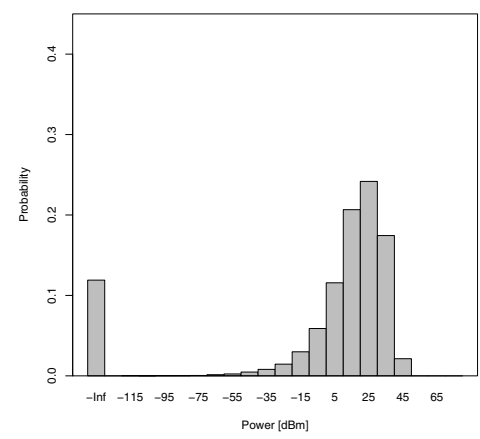

(b)

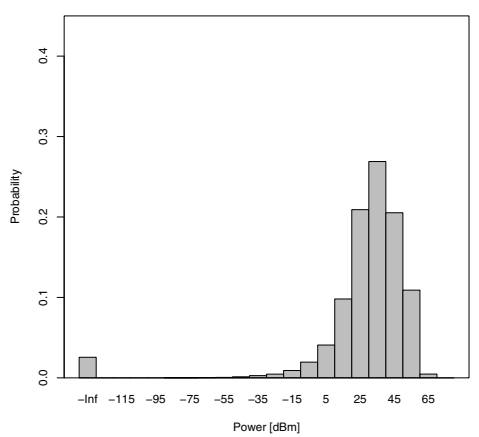

(c)

Fig. 6. The histograms of resulting transmit power summed over all the primary user channels for the random scenario. 
analytical model of the interference probability. The designed algorithm has been made quite conservative with respect of protecting primary systems. This can be seen through the approximations we have done in theoretical modeling, from which the algorithm is derived. This will lead to some losses in the spectrum reuse efficiency but will encourage license holders to share their frequencies.

We studied the performance of the algorithm in a simple scenario consisting of hexagonal cells to understand its behavior. Moreover, we compared the algorithm to an ideal algorithm where the positions of the active base stations are known in more realistic scenarios. Simulations showed that the proposed algorithm always respects the interference constraint and gives results that are close to the ideal ones in term of secondary satisfaction. We also highlighted the impact of knowing additional information such as the spectrum assignment to the cells and we showed the gains resulting from this additional information. We also identified the importance of knowing the traffic pattern of primary base stations to further improve the performance of the algorithm. The analysis of this is left for the future work.

\section{ACKNOWLEDGMENT}

We acknowledge a partial financial support from European Union through ARAGORN and FARAMIR projects. We also thank the financial support from Deutsche Forschungsgemeinschaft and RWTH Aachen University through UMIC-research centre. We would also like to thank Huawei Technologies CO., LTD for providing partial funding of this work through the CIRION project.

\section{REFERENCES}

[1] A. Ghasemi and E. S. Sousa, "Spectrum sensing in cognitive radio networks: the cooperation-processing tradeoff," Wireless Communications and Mobile Computing, vol. 7, no. 9, pp. 1049-1060, May 2007.

[2] R. Tandra, M. Mishra, and A. Sahai, "What is a spectrum hole and what does it take to recognize one?" Proceedings of the IEEE, vol. 97, no. 5, pp. 824-848, May 2009.

[3] S. M. Mishra, R. Tandra, and A. Sahai, "Coexistence with primary users of different scales," in the 2nd IEEE International Symposium on New Frontiers in Dynamic Spectrum Access Networks (DySPAN07), Dublin, 2007, pp. 158-167.
[4] E. G. Larsson and M. Skoglund, "Cognitive radio in a frequencyplanned environment: some basic limits," IEEE Transactions on Wireless Communications, vol. 7, no. 12, pp. 4800-4806, December 2008.

[5] A. O. Nasif and B. L. Mark, "Opportunistic spectrum sharing with multiple cochannel primary transmitters," IEEE Transactions on Wireless Communications, , vol. 8, no. 11, pp. 5702 - 5710, November 2009.

[6] "Second report and order and memorandum opinion and order," in ET Docket No. 04-186 and ET Docket No. 02-380, FCC 08-260, November 42008.

[7] M. Haddad, M. Debbah, and A. M. Hayar, "Distributed power allocation for cognitive radio," in the 9th International Symposium on Signal Processing and Its Applications (ISSPA 2007), Sharjah, United Arab Emirate, February 2007, pp. 1-4.

[8] L. Qian, J. Attia, X. Li, and Z. Gajic, "Joint power control and admission control for cdma cognitive radio networks," in the 15th IEEE Workshop on Local and Metropolitan Area Networks (LANMAN 2007), New Jersey, USA, June 2007.

[9] H. Zhu and J. Wang, "Chunk-based resource allocation in OFDMA systems - part I: chunk allocation," IEEE Transactions on Communications, vol. 57, no. 9, pp. 2734-2744, September 2009.

[10] A. Nasif and B. Mark, "Collaborative opportunistic spectrum access in the presence of multiple transmitters," in IEEE Global Telecommunications Conference (GLOBECOM 2008), 30 2008-Dec. 4 2008, pp. 1-5.

[11] J. Nasreddine, O. Sallent, J. Pérez-Romero, and R. Agustí, "Positioningbased framework for secondary spectrum usage," Physical Communication, vol. 1, no. 2, pp. 121-133, 2008.

[12] Y. Zhao, B. Le, and J. H. Reed, Cognitive Radio Technology. Elsevier, 2006, ch. Network Support: The Radio Environment Map, pp. 337-363.

[13] "IEEE standard for architectural building blocks enabling networkdevice distributed decision making for optimized radio resource usage in heterogeneous wireless access networks," IEEE Std 1900.4-2009, pp. C1-119, 272009.

[14] R. Chen, J.-M. Park, and J. H. Reed, "Defense against primary user emulation attacks in cognitive radio networks," IEEE Journal on Selected Areas in Communications, vol. 26, no. 1, pp. 25-37, Jan. 2008.

[15] J. Nasreddine, J. Pérez-Romero, O. Sallent, and R. Agustí, "A primary spectrum management solution facilitating secondary usage exploitation," in ICT-Mobile Summit, Stokholm-Sweden, 2008.

[16] T. Rappaport, Wireless Communications: Principles and Practice. Prentice-Hall, 2002.

[17] "Selection procedures for the choice of radio. transmission technologies of the umts (umts 30.03)," ETSI TR 101112 v3.2.0, April 1998.

[18] AG-Siemens, "TDD UE-UE interference simulations," TSG-RAN Working Group 4 (Radio meeting n26), 2003.

[19] http://www.t-mobiletowers.com/TowerSearch.aspx?mode=new [Last visited: 1st of February 2010]. 